\title{
Molecular Iodine: A Versatile Catalyst for the Synthesis of 2-Aryl-2,3-dihydroquinolin-4(1H)-ones
}

\author{
Liqiang Wu,' Bingxuan Niu, Weilin Li, and Fulin Yan \\ School of Pharmact, Ninxiang Medical Unwersitr, Ninxiang, Henan 453003. P. R. China. E-mall: whiq197toisohu.com \\ Received July 7, 2009, Accepted September 14, 2009
}

Key Words: 2-Ary 1-2,3-dilydroquinolin-4(1H)-ones. Iz. 2'-Aminochalcones, Solvent-free

2-Aryl-2.3-dihy droquinolin-4( $(1 H)$-ones are of considerable interest as they possess a wide range of pharmacological and therapeutic properties such as antitumor. ${ }^{1 a}$ anthelmintic activities. ${ }^{\mathrm{b}}$ In addition, they are also useful synthetic intermediates for various pharmaceuticals and active compounds. ${ }^{3}$ The formation of 2.3-dihydroquinolin-4( $1 H$-ones is generally' accomplished by isomerization of substituted 2 'aminochalcones in the presence of $\mathrm{NaOEt}^{3 \mathrm{ad}} \mathrm{H}_{3} \mathrm{PO}_{4}{ }^{3 \mathrm{~b}}$ montmorillonite. ${ }^{3 \mathrm{~b}}$ $\mathrm{InCl}_{3 .}{ }^{3 \mathrm{~d}}$ silica gel supported $\mathrm{TaBr}$ se $^{3 \mathrm{e}}$ silica gel supported

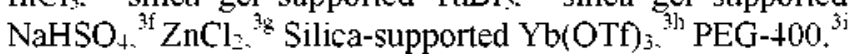
alumina supported- $\mathrm{CeCl}_{3} \cdot 7 \mathrm{H}_{2} \mathrm{O}-\mathrm{NaI}{ }^{3{ }^{3 j}}$ However. most of these procedures have signicant drawbacks such as long reaction times. low yields. harsh reaction conditions. tedious workup procedures. use of environmentally toxic reagents or media. Thus. there is still need of a simple and general procedure for synthesis of 2.3 -dilydroquinolin- $4(1 H)$-ones under mild conditions.

In recent years. the use of molecular iodine in organic synthesis has received considerable attention. Because of numerous advantages associated with this eco-friendly element. iodine has been explored as a powerful catalyst for various organic transformations. ${ }^{4}$ We now report a highly efficient procedure for the preparation of 2.3-dilydroquinolin- $4(1 H)$ ones using $I_{2}$ as an efficient and versatile catalyst under<smiles>CC=CC(=O)c1ccccc1N</smiles>
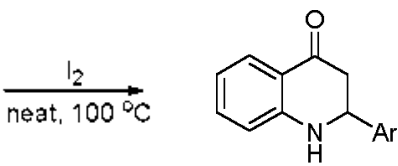

2
Scheme 1

Table 1. Temperature optimization for the synthesis of 2-phenv]-2,3dilyydroquinolin- $4(1 / H)$-one ${ }^{a}$

\begin{tabular}{cccc}
\hline Entry & Temp $/{ }^{\circ} \mathrm{C}$ & Time $/ \mathrm{h}$ & Yield/\% ${ }^{b}$ \\
\hline 1 & 60 & 6 & 61 \\
2 & 70 & 6 & 66 \\
3 & 80 & 5 & 72 \\
4 & 90 & 4 & 84 \\
5 & 100 & 3 & 92 \\
6 & 110 & 3 & 91 \\
7 & 120 & 3 & 92 \\
\hline
\end{tabular}

${ }^{a}$ Reaction conditions: (E)-1-(2-aminophenyl)-3-phenylprop-2-en-1-one (l mol): $\mathrm{I}_{2}(0.1 \mathrm{mmol})$; solvent-free. "Isolated yield. solvent-free conditions (Scheme 1)

Initially. to optinize the reaction temperature. the isomerization of $(E)$-1-(2-aminophenyl)-3-pheny lprop-2-en-1-one to the corresponding 2-pheny $1-2,3$-dihydroquinolin- $-4(1 H)$ ones was studied under solvent-free conditions in the presence of $10 \mathrm{~mol} \% \mathrm{I}_{\Sigma}$ at different temperatures. The results were summarized in Table 1 . As shown in Table 1 , the reaction at $100^{\circ} \mathrm{C}$ proceeded in highest yield.

The effect of amount of catalyst on the conversion and rate of the reaction was studied by varying the amount of $\mathrm{I}_{2}$ under solvent-free conditions at $100^{\circ} \mathrm{C}$ (Table 2). It was found that $10 \mathrm{~mol} \%$ of $\mathrm{I}_{2}$ was sufficient to carry out this reaction smoothly. An increase in the amount of $\mathrm{I}_{2}$ to more than $10 \mathrm{~mol} \%$ showed no substantial improvement in the yield. whereas the yield was reduced by decreasing the amount of $\mathrm{I}_{2}$ to $5 \mathrm{~mol} \%$.

Based on the optimized reaction conditions, a range of 2-arỵl-2.3-dilydroquinolin-4( $(1 H)$-ones (2) was șynthesized

Table 2. The amounts of catalyst optimization for the synthesis of 2-phenyl-2,3-dihydroquinolin-4( $1 H)$-one ${ }^{a}$

\begin{tabular}{cccc}
\hline Entry & Amounts of $\mathrm{I}_{2} / \mathrm{mol} \%$ & Time $/ \mathrm{h}$ & $\mathrm{Yield} / \%{ }^{\circ}$ \\
\hline 1 & 0 & 10 & 0 \\
2 & 5 & 5 & 71 \\
3 & 10 & 3 & 92 \\
4 & 15 & 3 & 92 \\
5 & 20 & 2 & 89 \\
6 & 25 & 2 & 92 \\
\hline
\end{tabular}

"Reaction conditions: (E)-1-(2-aminophenyl)-3-phenylprop-2-en-1-one $(1 \mathrm{mmol}): \mathrm{I}_{2}(0.1 \mathrm{mmol})$ : solvent-free: $100^{\circ} \mathrm{C}$. "Isolated vield.

Table 3. Synthesis of 2-aryl-2,3-dihydroquinolin-4( $1 H$ )-ones

\begin{tabular}{clccc}
\hline Entry & \multicolumn{1}{c}{ Ar } & Time/h & Product & Yield $/ \%$ \\
\hline 1 & $\mathrm{Ph}$ & $\mathbf{3}$ & $\mathbf{2 a}$ & 92 \\
2 & $4-\mathrm{NO}_{2} \mathrm{C}_{6} \mathrm{H}_{4}$ & 4 & $\mathbf{2 b}$ & 86 \\
3 & $4-\mathrm{ClC}_{6} \mathrm{H}_{4}$ & 4 & $\mathbf{2 c}$ & 85 \\
4 & $4-\mathrm{MeOC}_{6} \mathrm{H}_{4}$ & $\mathbf{3}$ & $\mathbf{2 d}$ & 92 \\
5 & $4-\mathrm{N}\left(\mathrm{Me}_{2} \mathrm{C}_{6} \mathrm{H}_{4}\right.$ & $\mathbf{3}$ & $\mathbf{2 e}$ & 93 \\
6 & $3-\mathrm{NO}_{2} \mathrm{C}_{6} \mathrm{H}_{4}$ & 4 & $\mathbf{2 f}$ & 89 \\
7 & $2-\mathrm{ClC} \mathrm{C}_{4}$ & 4 & $\mathbf{2 g}$ & 95 \\
8 & $2,6-\left(\mathrm{MeO}_{2} \mathrm{C}_{6} \mathrm{H}_{3}\right.$ & $\mathbf{3}$ & $\mathbf{2 h}$ & 88 \\
9 & $2,4-\left(\mathrm{Cl}_{2} \mathrm{C}_{6} \mathrm{H}_{3}\right.$ & $\mathbf{3}$ & $\mathbf{2 i}$ & 93 \\
10 & $3,4-(\mathrm{Cl})_{2} \mathrm{C}_{6} \mathrm{H}_{3}$ & 3 & $\mathbf{2 j}$ & 91 \\
\hline
\end{tabular}

"Isolated vield 
<smiles>Nc1ccccc1C(=O)/C=C/[Bi]</smiles><smiles>COC(=O)/C=N\[Al]/C=C/c1ccccc1OC</smiles><smiles>C=C</smiles><smiles>OC1=CC(Br)Nc2ccccc21</smiles><smiles>O=C1CC(Br)Nc2ccccc21</smiles>

Scheme 2

by the isomerization of 2 'aminochalcones (1). The reaction proceeded at $100^{\circ} \mathrm{C}$ within 4 hour in excellent yields after the addition of $10 \mathrm{~mol} \% \mathrm{I}$. (Table 3 ). The structures of the products were established from their spectral properties ( $\mathbb{R}$. ${ }^{\mathrm{H}} \mathrm{NMR}$. MS and elemental analy sis) and also by comparison with available literature data

In conclusion. we have described a convenient rolte to 2-ary 1-2,3-dihydroquinolin-4( $1 H)$-ones from 2'-aminochalcones under solvent-free conditions in the presence of $\mathrm{I}_{2}(10$ $\mathrm{mol} \%$ ) at $100^{\circ} \mathrm{C}$. The simple experimental procedure. solventfree reaction conditions, utilization of an inexpensive and readily available catalyst. short period of conversion and excellent yields are the advantages of the present method.

\section{Experimental Section}

A mixture of $2^{\circ}$-aminochalcones $(\mathrm{l} \mathrm{mmol})$ and $I_{z}(0.1$ mmol) was heated at $100{ }^{\circ} \mathrm{C}$ for the appropriate time (see Table 3). The reaction was monitored by TLC. After completion. the mixture was treated with aqueous $\mathrm{Na}_{2} \mathrm{~S}_{2} \mathrm{O}_{3}$ solution, extracted with diethyl ether $(3 \times 10 \mathrm{~mL})$. filtered and the solvent evaporated in vacuo. Products 2 were puried by silica gel column chromatography using hexane-diethyl acetate $(10: 1)$ as eluent.

2-(2,4-dichlomphenyl)-2,3-dihydroquinolin-t(1H)-one (2i). Yellow semi-solid. IR (cm $\left.{ }^{\mathrm{l}}\right): 3341(\mathrm{NH}), 1650(\mathrm{C}=\mathrm{O}) .{ }^{1} \mathrm{H}$ $\mathrm{NMR}\left(\mathrm{CDCl}_{3}, 400 \mathrm{MHz}\right) \delta 7.96(\mathrm{~d} .1 \mathrm{H}, J=1.4 \mathrm{~Hz}) .7 .81$ (dd, $\mathrm{IH}, J=1.0 .7 .6 \mathrm{~Hz}) .7 .62-7.50(\mathrm{~m}, 2 \mathrm{H}) .7 .26(\mathrm{dd} . \mathrm{IH}, J=1.4$. $8.0 \mathrm{~Hz}$ ) $6.80-6.65(\mathrm{~m} .2 \mathrm{H}) .+65$ (br s. $\mathrm{lH}) .5 .14$ (dd. $1 \mathrm{H} . J=$ 4.4, $13.6 \mathrm{~Hz}$ ). 2.88-2.82 (m, 2H); MS $m / z: 291\left(\mathrm{M}^{+}\right)$; Anal. calcd for $\mathrm{C}_{15} \mathrm{H}_{11} \mathrm{Cl}_{2} \mathrm{NO}: \mathrm{C} 61.67 . \mathrm{H} 3.79 . \mathrm{N} 4.79$ : found: $\mathrm{C}$ 61.47, H 3.88. N 4.85

2-(3,4-dichlomphenyl)-2,3-dihydroquinolin-4(1H)-one (2j). Yellow semi-solid. $\mathbb{R}\left(\mathrm{cm}^{\mathrm{l}}\right): 3312(\mathrm{NH}), 1662(\mathrm{C}=\mathrm{O}) .{ }^{1} \mathrm{H} N \mathrm{NR}$
$\left(\mathrm{CDCl}_{3} .400 \mathrm{MHz}\right) \delta 7.88(\mathrm{dd} .1 \mathrm{H} . J=1.0 .7 .6 \mathrm{~Hz}), 7.55-7.42$ (m. 2H). $7.23-7.02$ (m. 2H). 6.75-6.58 (m. 2H). 5.08 (dd. lH, $J=4.6,13.2 \mathrm{~Hz}), 4.58$ (br s, $1 \mathrm{H}), 2.85-2.79(\mathrm{~m}, 2 \mathrm{H})$ : MS $m / z$ : $291\left(\mathrm{M}^{+}\right)$: Anal calcd for $\mathrm{C}_{13} \mathrm{H}_{11} \mathrm{Cl}_{2} \mathrm{NO}: \mathrm{C} 61.67, \mathrm{H} 3.79, \mathrm{~N}$ 4.79: found: C 61.52. H 3.70. N 4.72.

Acknowledgments. We are pleased to acknowledge the financial support from Xinxiang Medical University.

\section{Reference}

1. (a) Xia, Y.; Yang, Z.-Y; Xia, P.; Bastow, K. F.; Tachibana, Y:; Kuo, S.C.; Hamel, E.; Hackl, T; Lee, K.-H. J. Hed Chem. 1998, 11,1155 : (b) Laliberte, R.: Campbell, D. J.: Bruderlein, F. Can. J. Pham Sci. 1967, 2, 37

2. (a) Prakash, O.; Kumar, D.; Saini, R. K.; Singh, S. P. Symh. Commun. 1994, 24, 2167 ; (b) Singh, O. V; Kapil. R. S. Sinth. Commm 1993, 23, 277

3. (a) Adrienne. L. T.: Laszlo. S. Swnth. Commm. 1987, 17, 1235: (b) Donnelly, I. A.: Fartell, D. F. J. Org. Chem. 1990, 55, 1757; (c) Varma, R. S. J. Heterocyclic Chem. 1999, 36, 1565; (d) Hemanth Kumar, K.; Muralidharan, D.; Penumal, P. T. Sinthesis 2004, 63: (e) Alumed, N.: van Lier, I. E. Tetrahedron Lett. 2006, 47,2725 . (f) Hemanth Kumar, K.: Perumal. P. T. Can. J. Chem. 2006, 84, 1079; (g) Jae, I. L.; Jir, J. H. J. Konew Chem. Soc. 2007, 51, 106; (h) Li, T.-T; Jin, L.-Y.; Yu, C.-M.; Su, W.-K. J. Chem. Res. 2009, 170: (i) Kumar, D.; Patel, G.; Mishra, B. G.; Vanma, R. S. Tetrahedron Lett. 2008, 19, 6974; (j) Ahmed, N.; van Lier, I. E. Tetrahedron Lett. 2007, $18,13$.

4. (a) Das, B.; Ravikanth, B; Ramu, R; Laxminarayana, K.; Rao, B. V. J. Mol. Catal A: Chemt 2006, 255, 74; (b) Kidwai, M.; Bansal, V.: Mothsra, P.: Sasena, S.: Somvanshi, R. K.; Dey, S.; Singh. T. P. J A lol Catal. A: Chem 2007, 268, 76; (c) Bhosale, R. S.; Magar, C. V; Solanke, K. S.; Mane, S. B.; Choudhary, S. S.; Pawar, R. P. Swht Conmun. 2007, 37, 4353; (d) Wu, T; Xia, H.-G.: Gao, K. Oig. Biomol. Chem. 2006, 4, 126. (e) Prajapati, D.: Gohain, M. Catal Lett. 2007, 119, 59. 